\title{
Decoherence of Electron Waves Due to Induced Charges Moving Through a Nearby Resistive Material
}

\author{
P. Sonnentag and F. Hasselbach \\ Institut für Angewandte Physik, Universität Tübingen, \\ Auf der Morgenstelle 10, D-72076 Tübingen, Germany
}

Received on 6 January, 2005

\begin{abstract}
An experiment in which decoherence, i.e. the transition from quantum to classical behaviour, can be studied in detail was proposed by Anglin and Zurek [1] and has now been realized. An electron beam in a biprism interferometer is split into two parts both of which travel over a plate made of a highly resistive material at the same, small height. The induced charges inside the plate move along with the beam electron, therefore a current results which encounters ohmic resistance. This process leads to a disturbance in the electron and phonon gas in the plate. As this disturbance is different for the two parts of the beam, entanglement between beam electron and plate is formed. The strength of decoherence, represented by the visibility of the interference fringes, varies as a function of two parameters, the height above the plate and the lateral separation of the beams. Allowing electrons of different height to reach the fluorescent screen successively, 'photos' of the quantum-classical border (continuous decrease of contrast with decreasing height above the plate) are built up.
\end{abstract}

\section{Introduction}

While the predictions of quantum theory were confirmed by experiments with incredible precision, a problem dating back (e.g., [2-4]) to the early days of quantum mechanics still survived: the incompatibility of the quantum mechanical superposition principle with our everyday experience of a 'classical' world in which, e.g., superpositions of macroscopically distinct states (like Schrödinger's cat [5]) are not observed and measurements give a definite result. A solution to this problem is given by decoherence. Decoherence is the emergence of classical features of a quantum system, resulting from its - in general irreversible - interaction with the environment [6-9].

Through this interaction, correlations are formed with the environment, i.e. we get entanglement of the system with its environment. Accordingly, it cannot be described by a (pure) quantum state, only the total system consisting of object plus environment is in a well-defined state. If one wants to know about the properties of the object, one has to take the partial trace of the state of the total system over the unobserved degrees of freedom, thereby getting a mixed state. If the object is in a superposition of states which become entangled with environment states being orthogonal to each other, the object seems to be in an incoherent mixture of these object states. So no interference between these states is left, classical behaviour emerges. If the environment states are not fully orthogonal, a fraction of interference remains.

In this paper, we present an experiment which vividly demonstrates the transition from quantum to classical. As a quantum system we use spatially split electron waves propagating over the surface of a resistive material (Fig. 1) which makes up the 'environment' in the present experiment. Using a microscopic object is necessary because for a macroscopic one there would always be strong decoherence due to the accessibility of many closely spaced energy levels. Utilisation of an electron has further advantages: 1) Electrons are the most simple, easy-to-handle, charged elementary particles without internal structure. 2) Their electromagnetic interaction is well understood and comparatively strong.

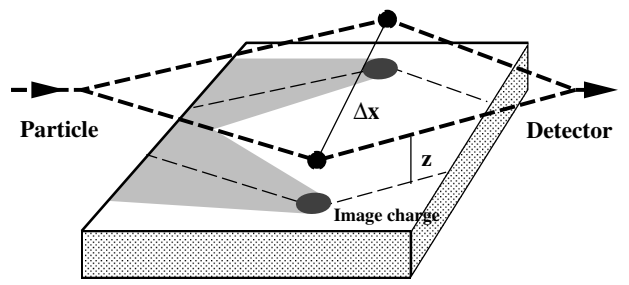

Figure 1. Sketch of the interference experiment on decoherence proposed by Anglin and Zurek [1].

In the resisitive material the induced charges caused by the electrons and the corresponding currents increase with decreasing height $z$ of the electron waves over the surface. Consequently, decoherence of the electron waves into classically behaving electrons is stronger for smaller heights over the plate than for larger distances from the plate. The decoherence of the electron waves is demonstrated by superimposing the two parts of the electron wave behind the resisitive plate and observing their ability to interfere. Electron waves propagating very near to the plate totally loose their possibility to interfere (complete dehoherence). With increasing distance of the electrons from the plate interference fringes with increasing contrast become visible. Another benefit of the present experiment is that by sending elec- 
trons in different heights through the 'interferometer' given in Fig. 1, the transition from quantum to classical becomes directly observable in a single 'photo': fringe contrast decreases continuously with decreasing distance $z$ from the resistive plate corresponding to increasing decoherence (Fig. 3 , rhs). The present experiment allows to study the degree of decoherence also as a function of a second parameter: By varying the lateral separation $\Delta x$ of the coherent beams one can easily test the dependence of decoherence on this additional parameter. As an interference device, an electron biprism was used in the experiment.

\section{The electron biprism interferome- ter}

The electron-optical bisprism [10] consists of a positively or negatively charged filament of less than $1 \mu \mathrm{m}$ in diameter, stretched between earthed electrodes located on both sides of it (Fig. 2). The incoming wavefront is divided into two parts when passing the biprism wire. If the filament is charged positively (negatively) they are deflected towards (apart from) each other. The deflection angle of the electron waves passing the field distribution in the vicinity of the filament (it is equivalent there to the field of a cylindrical capacitator) is the same for all paths irrespective of their distance from the center of the filament. In analogy to the Fresnel biprism in light optics, the divided wavefront seems to emerge from two virtual sources (dotted paths in Fig. 2). Since field-emitted electrons meet the spatial and temporal coherence conditions, interference fringes are formed in the region of superposition of the wavefronts. They are magnified electron-optically (not shown in Fig. 2) and observed on the screen of a channel plate image intensifier.

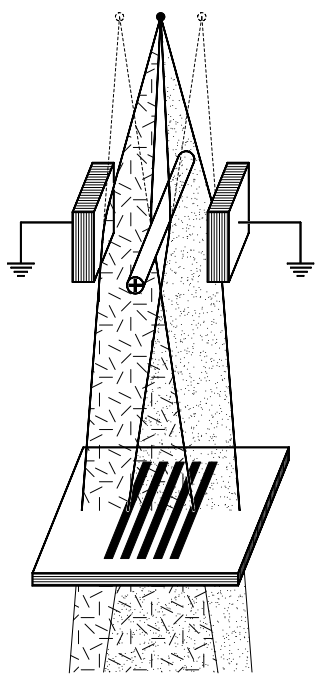

Figure 2. Scheme of the electron-optical biprism.

The first electron biprism interferometers and also today's electron holography instruments are modified elec- tron microscopes. Their most serious drawback is that due to their extremely high sensitivity to mechanical vibrations and to alternating electromagnetic fields, they can be operated only in special laboratories far from any traffic. In order to overcome these deficiencies a compact rigid electron biprism interferometer was developed [11]. In this type of interferometer, the electron-optical components are much smaller in dimension and in mass; they are, in general, of cylindrical shape with a diameter of $(28 \pm 0.01) \mathrm{mm}$ and are tightly screwed on two high-precision ceramic rods by means of braces. The arrangement is very rigid. Consequently, its mechanical eigenfrequency is raised to values far above the frequencies of floor vibrations and cannot be excited by these. Due to the construction principle, all elements are prealigned to the optical axis. Mechanical feedthroughs for alignment are not required. Fine alignment is achieved by electrostatic deflection elements and electromagnetic coils (with the latter, the image can be rotated). The whole interferometer can easily be surrounded by a highly effective tube of mu-metal with a shielding factor exceeding 5000. As a result, magnetic AC fields which would wash out the interference fringes are suppressed. As an electron source, a cold field emission gun is used necessitating an ultra-high vacuum environment. The electrons are accelerated by the field emission extraction voltage to their final energy in the range of $500-2500 \mathrm{eV}$ in a simple diode system consisting of a tungsten tip (cathode) on negative and an anode on earth potential.

This type of ruggedized electron interferometer has successfully been employed, e.g., to demonstrate the Sagnac effect with electrons $[12,13]$, to measure coherence length and energy spectra of electrons by means of Fourier spectroscopy [14, 15] using a Wien filter [16, 17], to prove electron antibunching [18], and to realize a biprism interferometer for $\mathrm{He}^{+}$ions [19] with their - compared to electrons extremely short wavelengths in the sub-pm range.

\section{Scheme of the decoherence experi- ment and experimental set-up}

For the decoherence experiment, the compact rigid interferometer (see Section 2) was utilized. In Fig. 3 the principle of the experimental set-up is given. Field-emitted electrons illuminate the negatively charged biprism filament which splits the wavefront into two parts and deflects them apart from each other. They are directed towards each other again by an electrostatic quadrupole (being negatively charged in the plane of the beams, see Fig. 3). Before the two beams meet, they travel over the resistive plate (a doped silicon wafer, $1 \mathrm{~cm}$ in length, with a resistivity $\rho \approx 1.5 \Omega \mathrm{cm}$ ) at the same, small height $z$, but with a lateral separation $\Delta x$. (By means of vertical deflection elements (see Fig. 4) in front of the 'decoherence plate', the rays are made parallel to the plate.) 


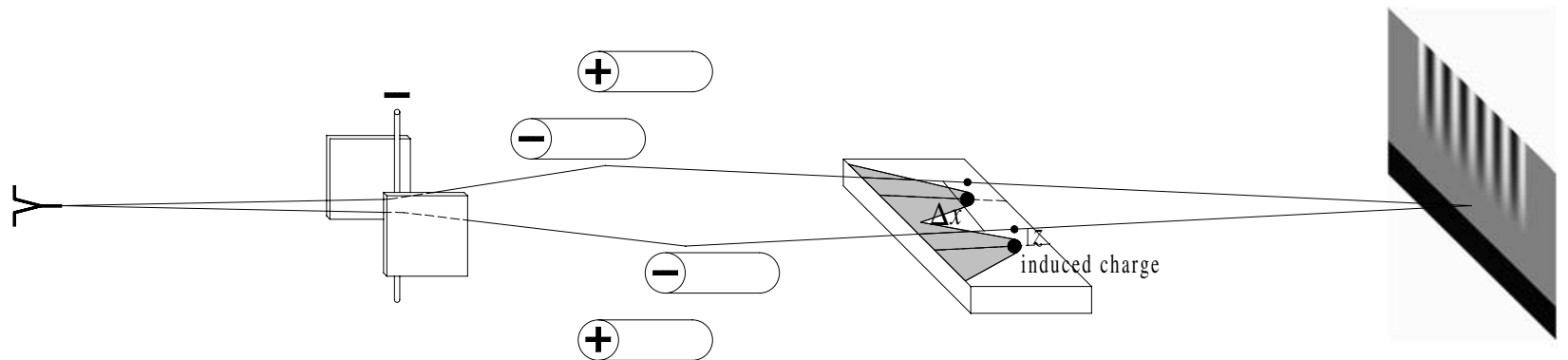

Figure 3. Sketch of the decoherence experiment. The two different paths of the electron over the resistive plate induce different disturbances (shaded regions) in the electron and phonon gas inside the plate. In the primary interference plane (rhs), interference fringes show less and less contrast the closer they are to the shadow of the plate.

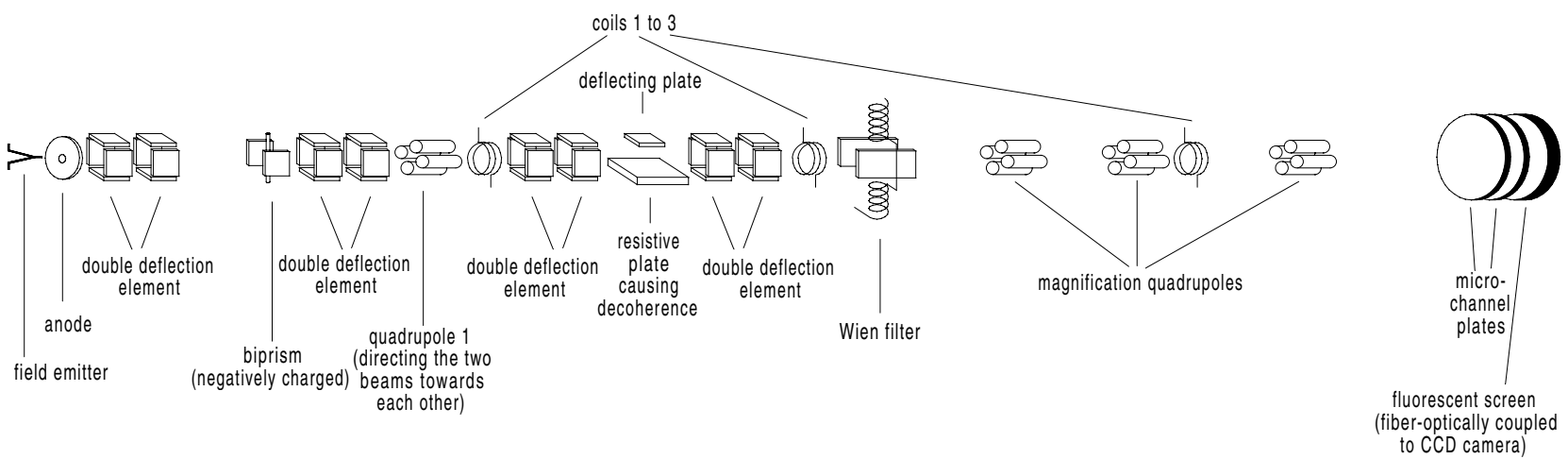

Figure 4. Schematical set-up of the compact rigid interferometer used in the decoherence experiment. (A corresponding photograph of the experiment is available from the authors upon request.)

As already mentioned, this gives rise to an interaction between the free electron and the plate: The electron induces a charge inside the plate, and this induced charge will move along with the beam electron. Therefore we get an electric current inside the plate, and the current encounters ohmic resistance. This leads to dissipation, there is Joule heating which quantum mechanically means that the state of the electron and phonon gas inside the plate is disturbed. The disturbance is different for the two parts of the beam. This can equivalently be interpreted either in terms of the availability of which-path information or in terms of entanglement of beam electron and plate. As the disturbance is irreversible, a record of the electron's path remains. So when the two parts of the beam are recombined, they are not fully capable of interference, and contrast of the interference fringes is reduced.

The interference pattern formed in the primary interference plane (where the two beams merge) is enlarged by electrostatic magnification quadrupoles. Its intensity is then amplified by a dual-stage channel plate image intensifier, transferred to a CCD camera by tapered fiber optics, and evaluated by an image processing system.

\section{Theoretical considerations}

Before the interaction of the beam electron with the electron and phonon gas inside the resistive plate, the state of the electron is a coherent superposition of the two parts of the beam,

$$
|\psi\rangle=(|l\rangle+|r\rangle) / \sqrt{2}
$$

where $|l\rangle$ resp. $|r\rangle$ denote the 'left' resp. 'right' part of the beam. The state of the electron and phonon gas before the interaction is certainly a mixed state. But for any of the pure states $\left|\phi_{0}\right\rangle$ into which this mixed state can be decomposed into, the interaction produces an entangled state of beam electron and electron $\&$ phonon gas:

$$
(|l\rangle+|r\rangle) / \sqrt{2} \otimes\left|\phi_{0}\right\rangle \rightarrow(|l\rangle \otimes|L\rangle+|r\rangle \otimes|R\rangle) / \sqrt{2}
$$

where $|L\rangle$ and $|R\rangle$ are the states of the electron \& phonon gas corresponding to the two parts of the beam electron.

The strength of the interaction and therefore the magnitude of the disturbance of the electron \& phonon gas certainly increases with decreasing height $z$ of the beams over the plate. Furthermore, the 'difference' between the two disturbed states of the electron \& phonon gas which correspond to the two different parts of the beam electron will increase with increasing lateral separation $\Delta x$ between the beams. From these simple considerations it is already clear that the absolute value of the scalar product $\langle L \mid R\rangle$ increases with increasing $\Delta x$ and with decreasing $z$. If we measure an observable affecting only the beam electron, interference (between states $|l\rangle$ and $|r\rangle$ ) is the more suppressed the smaller $\langle L \mid R\rangle$ is. So $|\langle L \mid R\rangle|$ gives the contrast of the interference fringes. In case of infite distance $z$ of beams to plate or in case of no separation between the beams over the plate, $\Delta x=0$, contrast will be maximum. 
Quasi-classical calculations [1] based on the rate of Joule heating in the plate,

$$
P=\frac{e^{2} \rho v^{2}}{16 \pi z^{3}}=-\frac{d}{d t}\left(\frac{m}{2} v^{2}\right)
$$

( $e$ being the elementary charge, $\rho$ the resistivity of the plate, and $v$ the velocity of the beam electron), and the relation between decoherence time $\tau_{\mathrm{d}}$ and relaxation time $\tau_{\mathrm{r}}=\frac{v}{|\dot{v}|}$,

$$
\tau_{\mathrm{d}}=\frac{\hbar^{2}}{m k_{\mathrm{B}} T(\Delta x)^{2}} \tau_{\mathrm{r}}
$$

( $m$ denoting the free electron mass, $k_{\mathrm{B}}$ the Boltzmann constant, and $T$ temperature), taken from a linear model $[20,21,22,23]$ lead to a decoherence time

$$
\tau_{\mathrm{d}}=\frac{4 h^{2} z^{3}}{\pi e^{2} k_{\mathrm{B}} T \rho(\Delta x)^{2}}
$$

which should be valid if $\rho$ and $\Delta x$ are not too large.

The contrast (visibility) of the electron interference fringes will then be

$$
\mathcal{V}=\exp \left(-\frac{t_{\text {flight }}}{\tau_{\mathrm{d}}}\right)
$$

where $t_{\text {flight }}$ is the time of flight of the beam electron over the plate. The expected course of fringe visibility as a function of height $z$ is shown in Fig. 5.

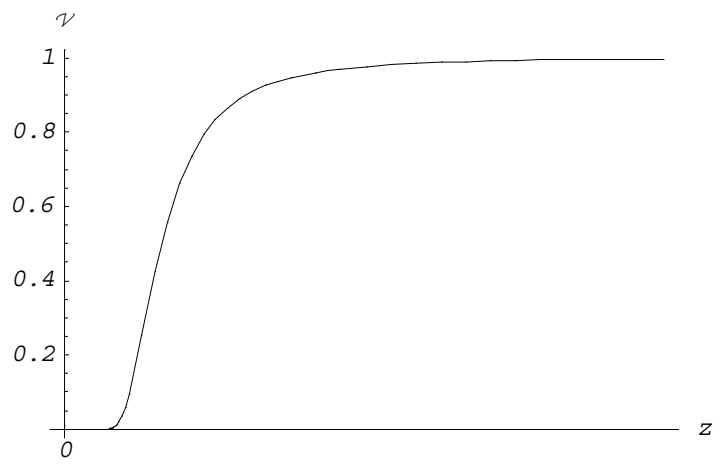

Figure 5. Expected course of fringe visiblity $\mathcal{V}$ as a function of height $z$ of beams over the plate.

There are other mechanisms which can also cause decoherence in this experiment: Vacuum fluctuations near a (perfect) conductor [24, 25] and emission of bremsstrahlung even in free space [26]. But both of these effects are much smaller than decoherence due to dissipation to the induced current in a poor conductor.

Stimulated by our experiment, Levinson [27] calculated the change in contrast due to vacuum fluctuations in the presence of dissipation.

\section{Results and discussion}

Figure 6 shows the interference fringes for electrons of $1.665 \mathrm{keV}$ of energy in heights above the resistive plate ranging from $z \approx 15 \mu \mathrm{m}$ to $z \approx 40 \mu \mathrm{m}$, for different values of $\Delta x$. (For heights below $15 \mu \mathrm{m}$, charging of dust particles on the plate leads to unwanted effects like deflection of the fringes. Therefore this region should not be used in the analysis of the data.) One can clearly see the decrease in visibility with decreasing height $z$. Also observable is the decrease in contrast with increasing lateral separation $\Delta x$. This decrease as a function of $\Delta x$ is not only caused by decoherence, but also by the fact that for smaller fringe spacing contrast is reduced due to lateral coherence. Quantitative analysis of the data is in progress and will be able to correct for the effect of lateral coherence by comparison with fringe contrast far away from the plate. It will also allow to compare the experimental results and the theoretical predictions of Anglin and Zurek [1] and of Levinson [27] and might give evidence if decoherence saturates [1] at a certain lateral separation $\Delta x$.

The pictures in Fig. 6 are images from the quantumclassical border showing directly the transition from the quantum regime (for large heights $z$, small lateral separations $\Delta x$ ) where there is full interference - i.e. an electron behaves totally like a wave - to the classical regime (for small $z$, large $\Delta x$ ) where (almost) full which-path information is available in principle - and an electron therefore behaves like a particle with a definite trajectory.

In order to be sure that the observed decrease in visibility is really caused by decoherence, other contrast-reducing effects had to be excluded. One of these effects might be that the longitudinal coherence condition is not fulfilled, i.e. that there is a wave packet shift - accumulated, e.g., in the electrostatic deflection elements where the two parts of the beam travel on different electric potential with different group velocities. Apart from the fact that one would expect this wave packet shift to be the same in all heights over the plate, one can eliminate it by using a Wien filter (crossed-field analyzer) $[16,17]$. The Wien filter consists of an electric and a magnetic field, both being perpendicular to the optical axis and to each other (Fig. 7 a, rhs). When the matching condition $E=v B$ between electric field $E$ and magnetic field $B$ is fulfilled for the main velocity component $v$ of the beam, there is neither a force on a beam electron (if it travels on the optical axis) nor does the Wien filter introduce a phase shift, but there is a wave packet shift due to the different electric potential for the two paths. By varying the excitation of the matched Wien filter, one can change the longitudinal distance between the interfering wave packets thus altering fringe visibility (Fig. 7 b). The maximum attainable contrast is the one corresponding to no resultant wave packet shift. Experimentally it was found that the contrast shown in Fig. 6 cannot be enhanced in any height by varying the Wien filter's excitation, so the loss of contrast in these pictures in not due to a wave packet shift.

At this point it is important to remark that the Wien filter itself does not introduce any irreversible decoherence [28]. This can be seen by the fact that the Wien filter can restore contrast to its maximum value (see Fig. 7 b). The reason for negligible decoherence in this case is that the probability for emission of a bremsstrahlung photon is extremely small $[26,29]$. 


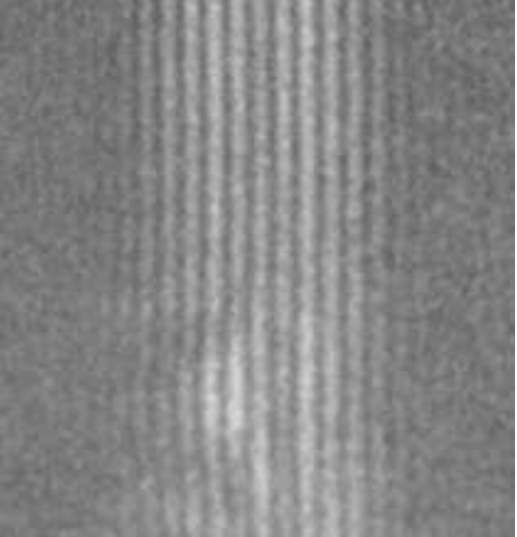

a)

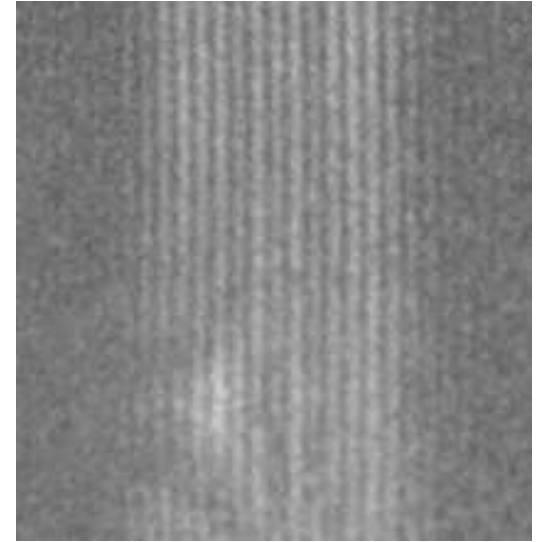

b)

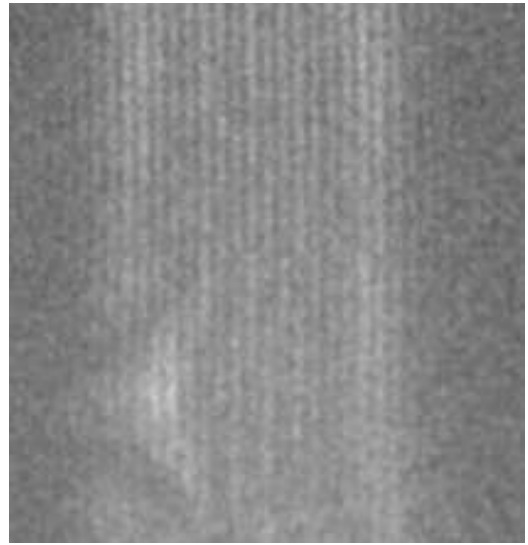

c)

Figure 6. Decoherence as a function of height $z$ of the beams over the plate for increasing lateral separations $\Delta x$ of the beams. The bottom of all pictures corresponds to a distance $z$ of $\sim 15 \mu \mathrm{m}$ from the plate, the top to $\sim 40 \mu \mathrm{m}$. The lateral separation $\Delta x$ is $\sim 11 \mu \mathrm{m}$ in a), $\sim 14 \mu \mathrm{m}$ in b), and $\sim 18 \mu \mathrm{m}$ in c). Both the increase of contrast (corresponding to a decrease in decoherence) from $z \approx 15 \mu \mathrm{m}$ to $z \approx 40 \mu \mathrm{m}$ and the decrease of contrast from a) to c) is clearly visible in the micrographs. The latter is caused by increasing decoherence and additionally by decreasing angular coherence with a larger lateral separation. The triangular structure which is visible near the bottom in all micrographs is due to charging of a dust particle on the biprism filament.
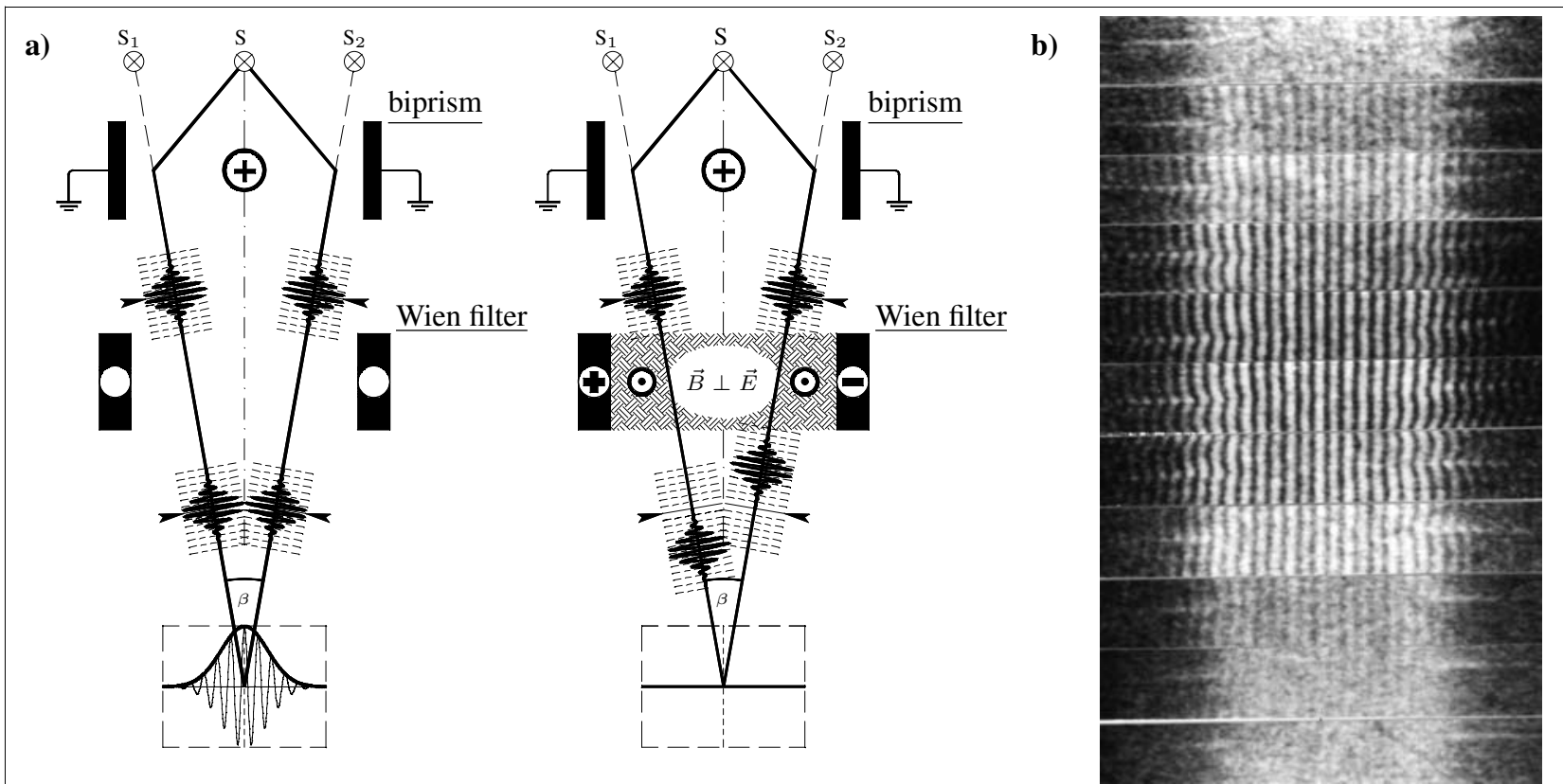

Figure 7. a) Electron biprism interferometer with Wien filter switched off (left) and Wien filter being excited (right): The wave packet shift exceeding the coherence length leads to the disappearance of the interference fringes. b) Restoration of contrast by a Wien filter: The longitudinal shift of the wave packets caused by electrostatic deflection elements (top) can be compensated (middle) and overcompensated (bottom) with the Wien filter [17]. The 11 micrographs correspond to 11 different excitations of the Wien filter, increasing from top to bottom.

Like for the case of a wave packet shift, all other conceivable effects which might reduce fringe visibility could be excluded as explanation of the loss of contrast observed in Fig. 6, leaving only decoherence as the reason.

Compared to other experiments on decoherence [30], the present one has several advantages: It is conceptually very simple, it realizes an actually ohmic environment (as it was supposed in the first theoretical treatments of decoherence), the quantum object is a single free elementary particle (no inner degrees of freedom are involved - due to the absence of magnetic fields, spin is irrelevant in the present experiment), the interaction with the environment is due to the electric field of a charge (thus giving us an idea why the charge superselection rule is so powerful), and we get real 'pictures' of the transition from quantum to classical.

\section{Acknowledgements}

We thank the Deutsche Forschungsgemeinschaft for fi- 
nancial support.

\section{References}

[1] J.R. Anglin, W.H. Zurek, in: Dark Matter in Cosmology Quantum Measurements - Experimental Gravitation : Proc. XXXIst Rencontres de Moriond, ed. by R. Ansari, Y. GiraudHéraud, J. Trân Thanh Vân (Editions Frontieres, Gif-surYvette, 1996), p. 263. quant-ph/9611049 v2.

J.R. Anglin, J.P. Paz, and W.H. Zurek, Phys. Rev. A 55, 4041 (1997).

[2] E. Schrödinger, Naturwissenschaften 14, 664 (1926).

[3] W. Heisenberg, Z. Phys. 43, 172 (1927).

[4] N. Bohr, Nature (London) 121, 580 (1928).

[5] E. Schrödinger, Naturwissenschaften 23, 807 (1935).

[6] H.D. Zeh, Found. Phys. 1, 69 (1970).

[7] W.H. Zurek, Phys. Rev. D 24, 1516 (1981).

[8] E. Joos et al., Decoherence and the Appearance of a Classical World in Quantum Theory, (Springer, Berlin, 2003).

[9] W.H. Zurek, Rev. Mod. Phys. 75, 715 (2003).

[10] G. Möllenstedt, H. Düker, Z. Phys. 145, 377 (1956).

[11] F. Hasselbach, Z. Phys. B 71, 443 (1988).

[12] F. Hasselbach, M. Nicklaus, Phys. Rev. A 48, 143 (1993).

[13] R. Neutze, F. Hasselbach, Phys. Rev. A 58, 557 (1998).

[14] F. Hasselbach, A. Schäfer, and H. Wachendorfer, Nucl. Instrum. Methods Phys. Res., Sect. A 363, 232 (1995).

[15] P. Sonnentag, H. Kiesel, and F. Hasselbach, Micron 31, 451 (2000)
[16] G. Möllenstedt, G. Wohland, in: Proc. Seventh Europ. Congr Electron Microscopy, ed. by P. Brederoo, G. Boom (North Holland, Amsterdam, 1980), p. 28.

[17] M. Nicklaus, F. Hasselbach, Phys. Rev. A 48, 152 (1993).

[18] H. Kiesel, A. Renz, and F. Hasselbach, Nature (London) 418, 392 (2002).

[19] F. Hasselbach, U. Maier, in: Quantum Coherence and Decoherence : Proc. ISQM-Tokyo '98, ed. by Y.A. Ono, K. Fujikawa (Elsevier, Amsterdam, 1999), p. 299.

[20] W.H. Zurek, in: Frontiers of Nonequilibrium Statistical Physics, ed. by G.T. Moore, M.O. Scully (Plenum, New York, 1986), p. 145.

[21] J.P. Paz, S. Habib, and W.H. Zurek, Phys. Rev. D 47, 488 (1993)

[22] E. Joos, in: [8], Chapter 3.

[23] H.-P. Breuer, F. Petruccione, The Theory of Open Quantum Systems (Oxford Univ. Press, Oxford, 2002), Chapter 4.3.

[24] L.H. Ford, Phys. Rev. D 47, 5571 (1993).

[25] F.D. Mazzitelli, J.P. Paz, and A. Villanueva, Phys. Rev. A 68 , 062106 (2003).

[26] H.-P. Breuer, F. Petruccione, Phys. Rev. A 63, 032102 (2001)

[27] Y. Levinson, J. Phys. A 37, 3003 (2004).

[28] F. Hasselbach, H. Kiesel, P. Sonnentag, in: Decoherence: Theoretical, Experimental, and Conceptual Problems, ed. by P. Blanchard et al. (Springer, Berlin, 2000), p. 201.

[29] C. Kiefer, in: [8], Chapter 4.

[30] L. Hackermüller et al., Nature (London) 427, 711 (2004), and references therein. 